\title{
ATTACHMENT OF THE CAP TO THE CENTRAL MICROTUBULES OF TETRAHYMENA CILIA
}

\author{
WILLIAM L. DENTLER
}

Department of Physiology and Cell Biology, Center For Biomedical Research, 2095 Avenue A, Campus West, University of Kansas, Lawrence, KS 66044, U.S.A.

\begin{abstract}
S U M M A R Y
The central microtubule cap is bound to the ends of the two central microtubules in Tetrahymena themophila cilia by plug-shaped structures similar in appearance to the distal filament plugs attached to the ends of the A-microtubules. The caps have been separated from the microtubules and are composed of a bead, two plates, and two peg-like plugs to which the microtubules are attached. The structure of the cap is discussed in relation to the directionality of microtubule assembly in vivo.
\end{abstract}

\section{INTRODUCTION}

The distal ends of both the central and outer doublet A-microtubules are capped by distinct structures in virtually all cilia and flagella (Dentler, 1981; Dentler \& LeCluyse, 1982b). The central microtubule cap is bound to the ends of the two central microtubules and is composed of two plates, bound to the microtubules, and a bead that is bound to the membrane. Initial studies of the cap suggested that the microtubules terminated evenly on the proximal plate (Dentler \& Rosenbaum, 1977; Sale \& Satir, 1977). The end of each of the nine A-microtubules is attached to the distal filament plug, composed of a carrot-shaped plug bound to the inner wall of each A-microtubule (Dentler, 1980a) and a filamentous portion bound to the membrane, as in Tetrahymena and Chlamydomonas (Dentler, 1980a), or to the central microtubule cap, in tracheal cilia (Dentler \& LeCluyse, 1982b).

Initial studies revealed that both the central microtubule cap and distal filament plugs were bound to the ends of both growing and fully grown microtubules in Tetrahymena cilia and Chlamydomonas flagella but that the cap was more tightly bound to the central microtubules than were the distal filament plugs to the A-microtubules (Dentler \& Rosenbaum, 1977; Dentler, 1980a). Since autoradiographic studies had shown that most newly synthesized proteins were incorporated into the distal ends of the A-microtubules (Rosenbaum, Moulder \& Ringo, 1969; Witman, 1975) and since the proximal ends of the central microtubules were not continuous with basal body microtubules (as were the outer doublets) it was suggested that the central microtubules might assemble at their uncapped proximal ends (Dentler \& Rosenbaum, 1977; Dentler, 1980a). Since the intrinsic polarity of the central and doublet microtubules is the same (Euteneur \& McIntosh, 1981), tubulin would have to add to the minus ends of the central microtubules and to the plus ends of the 
doublets during ciliary assembly. Although microtubules have been shown to assemble onto the proximal (but not distal) ends of capped central microtubules in tracheal cilia (Dentler \& LeCluyse, 1982a), the site of central microtubule assembly in vivo has not been determined by direct experiment.

To determine the site of central microtubule assembly and to determine the role of the capping structures in assembly it will be necessary to characterize the linkage of the cap to the central microtubules, to isolate the caps and test for their ability to nucleate microtubules with specific polarity, and to determine the directionality of central microtubule assembly in vivo using pulse-labelling and autoradiography. The goals of the present study were to reveal the structures that link the central microtubules to the cap and to present preliminary observations on the structure of caps that were released from ciliary microtubules.

\section{MATERIALS AND METHODS}

Tetrahymena thermophila (strain SB711) was grown on $2 \%$ proteose peptone as previously described (Dentler, 1980a) and cilia were isolated from 1.5-litre cultures using a modification of the dibucaine procedure (Dentler, 1979). Isolated cilia were demembranated by two washes in $0.1 \%$ Nonidet P-40 in PMEDPS (100 mm-PIPES (piperazine- $N, N^{\prime \prime}$-bis(2-ethanesulphonic acid)), $5 \mathrm{~mm}$ $\mathrm{MgSO}_{+}, \quad 0 \cdot 1 \mathrm{~mm}-\mathrm{EGTA}$ (ethyleneglycol-bis(amino-ethylether) $N^{N}, N^{\prime \prime}$-tetraacetic acid), $1 \mathrm{~mm}$ dithiothreitol, $0.3 \mathrm{~mm}$-PMSF (phenylmethylsulphonyl fluoride), $0.25 \mathrm{~m}$-sucrose, $\mathrm{pH} 6.9$ ) and washed once in PMEDPS without detergent. To release central microtubule caps, axonemes were washed with PMEDPS with increasing concentrations of $\mathrm{NaCl}$. Both whole cilia and caps were negatively stained with 1 \% uranyl acetate and were viewed and photographed using a Philips EM 300.

\section{RESULTS}

The central microtubule cap in intact Tetrahymena axonemes appeared similar to that in Chlamydomonas flagella (Fig. 1A, Dentler \& Rosenbaum, 1977; Dentler,

Fig. 1A. Negatively stained Tetrahymena cilium showing the central microtubule cap ( $c m c$ ) attached to the central microtubules $(c p)$ and the distal filaments $(D)$ bound to each of the A-microtubules of the outer doublets $(a) . \times 200000$.

B-F. Series of micrographs showing central microtubule caps partially released from the central microtubules when axonemes are incubated in increasing concentrations of salt. The prominent bead ( $b$ in $B$ and 1$)$ that links the central pair with the membrane and the two plates (arrows in $\mathrm{B}, \mathrm{D}, \mathrm{k}$ ) are retained throughout the extraction procedure. As the caps are released small plugs $(p)$ linking the caps to the microtubules are revealed. In addition to the plugs, a small bead structure is seen to lie between the two microtubules (arrowhead in $c$ ). The bead hangs like a pendent from the proximal plate of the cap. Since the distal ends of the central microtubules immediately beneath the cap, are tightly apposed (see brackets in $A$ and $B$ ), the bead may serve to link these microtubules together and provide additional structural support for the attachment of the two microtubules bound to cap. $\times 200000$.

G. Cap in which the beaded portion of the cap is released and the lower plate is firmly attached to the central microtubules. A plug can be seen to extend for a very short distance into the lumen of each microtubules. $\times 200000$.

$\mathrm{H}-\mathrm{M}$. Caps that have been released from the central microtubules and, in $\mathrm{I}-\mathrm{M}$, were separated from microtubules by differential centrifugation. The caps retain most of the structures observed in partially extracted cilia $(A-F)$ although the plug structures (arrowheads in $\mathrm{I}-\mathrm{M}$ ) attached to the proximal plate are somewhat more extended than they are in intact microtubules. $\times 200000$. 
Central microtubular cap in Tetrahymena

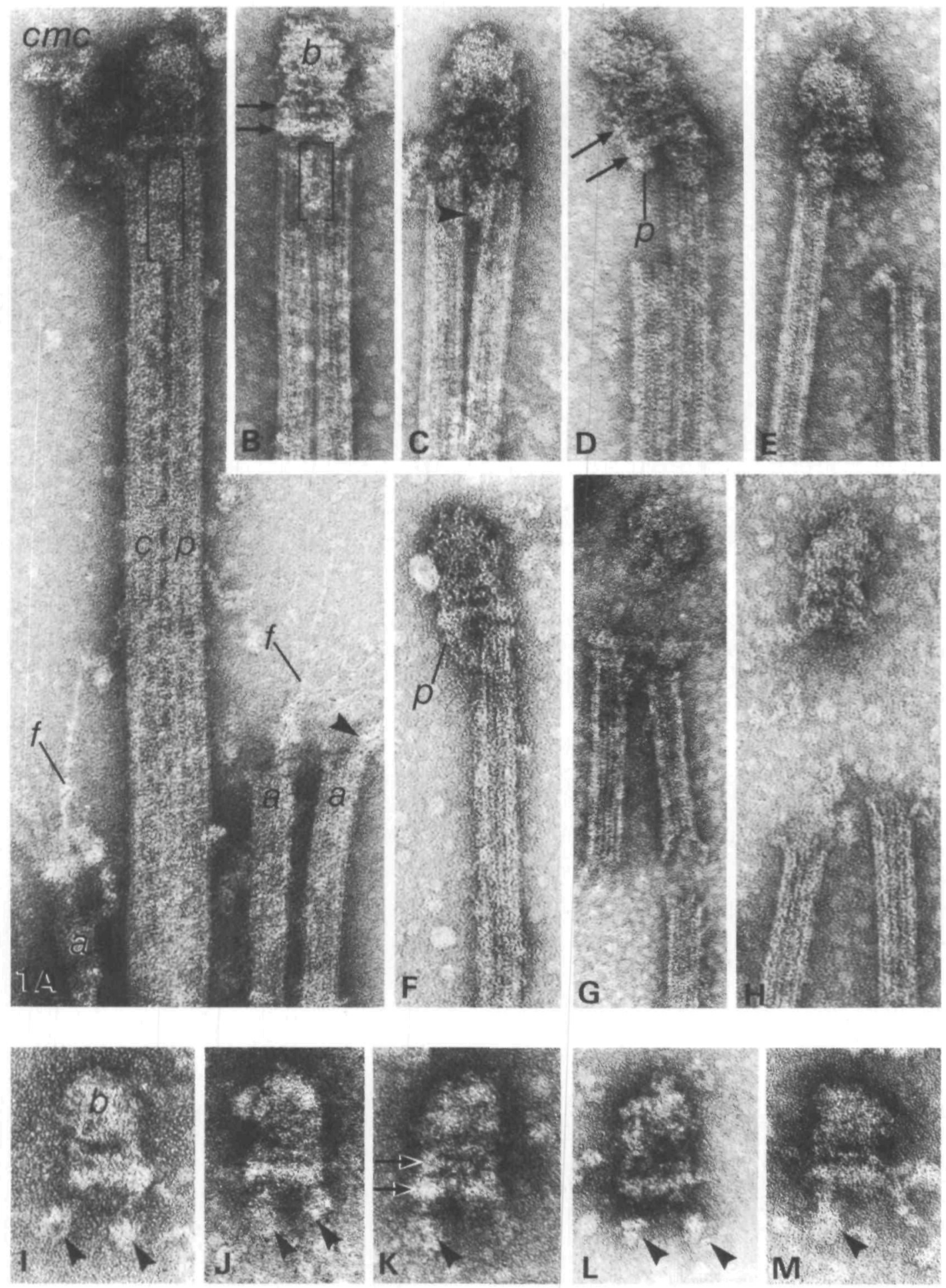

Fig. 1 
$1980 a$; Sale \& Satir, 1977) and was composed of a $43 \mathrm{~nm}$ diameter spherical bead that attached to two $53 \mathrm{~nm}$ diameter plates. The distal plate (attached to the bead) was $13 \mathrm{~nm}$ thick and frequently appeared to be composed of two thinner plates (Fig. 1 $\mathrm{B}$, D, F). The proximal plate was $9 \mathrm{~nm}$ thick and was attached to the central microtubules. In intact axonemes the sites of microtubule attachment were somewhat thicker than the rest of the plate (Fig. 1A, B, G).

When the caps were released from the microtubules during extraction with $\mathrm{NaCl}$, these thickenings were found to be connected to plug structures inserted into the lumen of each central microtubule (Fig. 1D, E, F). The plugs were quite short and stubby when initially released from the microtubules (Fig. ID, E, H) but often elongated as the period of salt extraction was increased to release the caps from all axonemes (compare Fig. 1 I-M with D, E, H). The centre-to-centre spacing of the two plugs attached to completely released caps was $40 \mathrm{~nm}$ but, in intact axonemes, was only $25 \mathrm{~nm}$, the same centre-to-centre spacing as that of the two central microtubules.

The central microtubules in intact axonemes were tightly joined together near their distal tips (see brackets in Fig. 1A, B). As the caps were released from the microtubules, a small $10 \mathrm{~nm}$ diameter bead was revealed lying between the two microtubules (Fig. 1C). The bead was attached at or near the sites of plug attachment on the proximal plate and looked like a pendant hung on a necklace. The $10 \mathrm{~nm}$ bead was frequently observed to be attached to the proximal plate in fully released caps and was more labile than the plugs because it often disappeared during salt extraction of the axonemes.

\section{DISCUSSION}

The attachment of the caps to the central microtubules is structurally similar to that of the attachment of the distal filament plugs to the A-microtubules of each outer doublet. Each capping structure is composed of a plug inserted into the lumen of the microtubule and a portion that attaches to the membrane (as in the central microtubule caps and the distal filaments of Tetrahymena and Chlamydomonas; Dentler, 1980a) or to the central microtubule cap (as in cilia from mammalian and avian trachea and in amphibian palates; Dentler \& LeCluyse, 1982b; LeCluyse \& Dentler, 1983). Although best visualized in negatively stained cilia, the plugs can also be seen in cross-sections of cilia as small 'dots' in the lumens of $A$ and central microtubules near their distal tips (see Dentler \& LeCluyse, 1982b). The plugs in the $A$ and central microtubules are not, however, identical. Plugs in the A-microtubules of Tetrahymena are long tapered structures, while those in Tetrahymena central microtubules are short and stubby. Detailed comparisons of plug structure in tracheal or palate cilia have not been made.

In addition to the two plugs, a bead structure hung from the proximal plate of the cap. The bead lay between the ends of the central microtubules and appeared to link them together in intact axonemes. When the caps were partially released from the central microtubules the beads did not appear to be attached directly to the surface of either microtubule, which suggests that the function of the bead may not be to link 
the microtubules together. Ringo (1967) initially reported that a small dense plate similar to the Tetrahymena bead structure lay between the two central microtubules immediately beneath the cap in Chlamydomonas and Dentler \& Rosenbaum (1977) reported that the distal ends of the central microtubules in Chlamydomonas are wrapped together by a thin filament. It remains to be determined whether the structures interposed between the ends of central microtubules in Tetrahymena and Chlamydomonas are linking structures, whether they are similar to each other and whether they are common to all cilia and flagella.

What is the function of the central microtubule cap and distal filament plugs? It is most likely that they are involved with either motility or microtubule assembly. However, demembranated cilia and flagella can be reactivated to beat and microtubules to slide with caps that are either present or released (unpublished observations), so the capping structures are not essential for the motility of demembranated and reactivated cilia. The cap and plug complexes in tracheal cilia may restrict microtubule sliding (Dentler \& LeCluyse, 1982b) but sliding can occur in tracheal cilia that lack caps (Dentler, 1980b; Dirkson \& Zeira, 1981). Since the caps link the microtubule ends to the membrane it is possible that they protect these ends from abrasion by the membrane during ciliary bending but other motility-related functions are unknown.

Several types of evidence suggest that the capping structures are involved in microtubule assembly. Previous studies of Chlamydomonas, Tetrahymena and tracheal cilia (Dentler, 1981; Dentler \& LeCluyse, preliminary data) revealed that the central microtubule cap and distal filament plugs were formed during the first $2 \mu \mathrm{m}$ of ciliary growth and that they remained associated with the microtubule ends in growing and in fully grown cilia. In addition to being associated with the microtubule ends, examination of capping structures in epithelial cilia in acoel turbellarians (Tyler, 1979), the frog Bombina and the toad Xenopus (LeCluyse \& Dentler, 1983) revealed that specific caps can be precisely positioned on both central and individual outer doublet A-microtubules. This precise positioning was correlated with individual doublet and central microtubules that have characteristic lengths.

Studies of demembranated cilia indicate that the capping structures are tightly bound to the microtubule ends. The plugs in the $A$ and central microtubules in tracheal cilia remain attached to the microtubule ends after demembranation and reactivation of ciliary beating (Dentler \& LeCluyse, 1982b). If the associations between the caps and microtubules were weak, the plugs would have been expected to have been pulled free of the microtubules due to forces generated by microtubule sliding.

In addition to resisting microtubule sliding forces, the caps are so tightly bound to the central microtubules of Chlamydomonas and tracheal cilia that they blocked the addition of brain microtubule protein to these microtubules in vitro (Dentler \& Rosenbaum, 1977; Dentler \& LeCluyse, 1982a). Since these in vitro studies also revealed that microtubule assembly occurred at the proximal ends of the central microtubules, which are not capped, it was suggested that the central microtubules assemble in vivo at their proximal ends. On the other hand, studies of demembranated tracheal cilia revealed that the plugs also block the addition of brain tubulin onto the 
A-microtubules in vitro (Dentler \& LeCluyse, 1982a). Since autoradiographic studies have shown that the site of A-microtubule assembly in vivo is at the distal tip (at or near the site of the plugs) (Rosenbaumet al. 1969; Witman, 1975), the plugs must not completely block microtubule assembly in vivo. Since the results reported here showed that the central microtubule caps are bound to the central microtubules by plug structures that are similar at least in appearance to the distal filament plugs, it is not unreasonable to suggest that the central microtubules also assemble at their distal (capped) ends. Further studies of the sites of central microtubule assembly in regenerating flagella will be necessary to determine the sites of central microtubule assembly.

Since the plug structures are present at the apparent sites of microtubule assembly in vivo throughout ciliary growth and since in vitro studies have shown that they are tightly bound to the microtubule ends, a scaffolding mechanism must exist that permits the plugs on the A-microtubules and, possibly, those attached to the central microtubules to be moved up the growing cilium at the site of microtubule assembly. The presence of plug structures within the lumens of both $A$ and central microtubules suggests that similar mechanisms might regulate the associations of plugs with microtubules. Since the plugs are at the growing ends of the microtubules it is possible that they may be involved in the regulation of microtubule length, possibly by regulating access of tubulin to the microtubule end (see Dentler $\&$ LeCluyse, 1982b). Recent studies by Brunke, Collis \& Weeks (1982) and by L'Hernault \& Rosenbaum (1982) have shown that tubulin is post-translationally modified as it is assembled into Chlamydomonas fagellar microtubules and it is possible that one of the roles of the capping structures is to carry out this modification.

Since it is now apparent that the central microtubule caps can be released from the cilia it should be possible to study the associations between these capping structures and tubulin or microtubules. Moreover, the isolation and characterization of the capping structures may lead to the investigation of the ends of cytoplasmic microtubules to determine if similar capping structures link the ends of these microtubules to membranes, kinetochores, or other cytoplasmic structures. Although capping structures have not been observed to be associated with cytoplasmic microtubules, electron-dense material has been found between the plasma membrane and the ends of microtubules associated with Tetrahymena contractile vacuoles (Allen \& Wolf, 1979; Scott \& Hufnagel, 1983) and with secretory cells in planarians (Dentler, unpublished data). Characterization of this dense material and its comparison with proteins associated with the plugs in ciliary microtubules will depend on the successful isolation and characterization of the ciliary microtubule capping structures.

I thank Dr E. Orias for donation of the mucus-free Tetrahymena strain used in these experiments. This work was supported by grants from the N.I.H. (AM21672 and GM32556) and by grants to the Center for Biomedical Research, University of Kansas.

\section{REFERENCES}

Allen, R. D. \& Wolf, R. W. (1979). Membrane recycling at the cytoproct of Tetrahymena.J. Cell Sa. 35, 217-227. 
Brunke, K. J., Collis, P. S. \& Weeks, D. P. (1982). Posttranslational modification of tubulin dependent on organelle assembly. Nature, Lond. 297, 516-518.

Dentler, W. L. (1979). Microtubule-membrane interactions in cilia. I. Isolation and characterization of Tetrahymena ciliary membranes. F. Cell Biol. 84, 364-380.

Dentler, W. L. (1980a). Structures linking the tips of ciliary and flagellar microtubules to the membrane. J. Cell Sci. 42, 207-220.

Dentler, W. L. (1980b). The central and outer doublet microtubules are attached to a cap in mammalian tracheal cilia. J. Cell Biol. 87, 36a.

Dentler, W. L. (1981). Microtubule-membrane interactions in cilia and flagella. Int. Rev. Cytol. $72,1-47$.

Dentler, W. L. \& LeCluyse, E. L. (1982a). The effects of structures attached to the tips of tracheal ciliary microtubules on the nucleation of microtubule assembly in ertro. Cell Motil. (suppl.) 1, 13-18.

Dentler, W. L. \& LeCluyse, E. L. (1982b). Microtubule capping structures at the tips of tracheal cilia: Evidence for their firm attachment during ciliary bend formation and the restriction of microtubule sliding. Cell Motil. 2, 549-572.

Dentler, W. L. \& Rosenbaum, J. L. (1977). Flagellar elongation and shortening in Chlamydomonas. III. Structures attached to the tips of flagellar microtubules and their relationship to the directionality of flagellar microtubule assembly. I. Cell Biol. 74, 747-759.

Dirksen, E. R. \& ZeIRA, M. (1981). Microtubule sliding in cilia of the rabbit trachea and oviduct. Cell Motil. 1, 372-376.

Euteneur, U. \& McIntosh, J. R. (1981). Polarity of some motility-related microtubules. Proc. natn. Acad. Sci. U.S.A. 78, 372-376.

LeCluyse, E. L. \& Dentler, W. L. (1983). Asymmetric microtubule capping structures in frog palate cilia. J. Ultrastruct. Res. (In'press).

L'Hernault, S. W. \& Rosenbaum, J. L. (1982). Reversible post-translational modification of $\alpha$-tubulin is coupled to flagellar assembly and disassembly in Chlamydomonas. 7. Cell Biol. 95 , $342 \mathrm{a}$.

Ringo, D. L. (1967). Flagellar motion and fine structure of the flagellar apparatus in Chlamydomonas. 7. Cell Biol. 33, 543-571.

Rosenbaum, J. L., Moulder, J. \& Ringo, D. L. (1969). Flagellar elongation and shortening in Chlamydomonas. I. The use of cycloheximide and colchicine to study the synthesis and assembly of flagellar proteins. J. Cell Biol. 34, 600-619.

Sale, W. S. \& SatiR, P. (1977). The termination of the central microtubules from the cilia of Tetrahymena pynformis. Cell Biol. Int. Rep. 1, 45-49.

SCOTt, S. M. \& HufNagel, L. A. (1983). The effect of concanavalin A on egestion of food vacuoles in Tetrahymena. Expl Cell Res. 144, 429-441.

TYLER, S. (1979). Distinctive features of cilia in metazoans and their significance for systematics. Tissue Eீ Cell 11, 385-400.

W ITMan, G. B. (1975). The site of in vivo assembly of flagellar microtubules. Annls N.Y. Acad. Sci. 253, 178-191.

(Received 28 May 1983-Accepled, in revised form, 12 September 1983) 
